Recepción: 08 / 03 / 2017

Aceptación: 17 / 05 / 2017

Publicación: 15 / 07 / 2017 (c) (P)

Artículo de investigación

\title{
Factores de riesgo asociados a trastornos temporomandibulares
}

\author{
Risk factors associated with temporomandibular disorders
}

Fatores de risco associados a distúrbios temporomandibulares

\author{
Juan A. Oliveira del Río ${ }^{\text {I }}$ \\ juanoliveiradelrio@ hotmail.com \\ Miguel Carrasco-Sierra II \\ miguelcsierra@gmail.com \\ Alba M. Mendoza-Castro II \\ albacastrom@gmail.com
}

Correspondencia: juanoliveiradelrio@hotmail.com

\begin{abstract}
I Doctor en Ciencias Odontológicas, Docente en la Universidad Laica Eloy Alfaro de Manabí, Manta, Ecuador.

II Master en Atención de Urgencias en Estomatología, Especialista de Primer Grado en Prótesis Estomatológica, Doctor en Estamología, Docente en la Universidad Laica Eloy Alfaro de Manabí, Manta, Ecuador.
\end{abstract}

III Magister en Gerencia de Salud para el Desarrollo Local, Odontóloga Especialista en Endodoncia, Docente en la Universidad Laica Eloy Alfaro de Manabí, Manta, Ecuador. 


\section{Resumen}

Son muy diversos los factores de riesgo de los trastornos temporomandibulares, por lo que debe hacerse un análisis pormenorizado de cada caso, para evaluar la posible concomitancia de varios de ellos, para darle el mayor peso al posible factor etiológico y prestar atención a los que se hagan perdurables o agravantes. Se realizó un estudio observacional, descriptivo, de corte transversal con vista a identificar los factores de riesgo asociados a los trastornos temporomandibulares. El universo estuvo comprendido por 70 pacientes que acudieron a la consulta en el momento de la investigación. Como principales variables analizadas figuraron: edad, sexo, factores predisponentes, factores precipitantes, enfermedades sistémicas. En la serie se obtuvo que del total de pacientes, 41 pertenecían al sexo femenino, para un 58.5\%. El grupo de edades de 40-49 años estuvo representado por 35 pacientes para un 50.0\%. En el $92.4 \%$ se observó bruxismo, en el 91.4\% masticación unilateral en tanto que el 92.8\% presentó dientes ausentes no restituidos.

Palabras clave: trastornos temporomandibulares; factores de riesgo; articulación temporomandibular.

\section{Abstract}

The risk factors of temporomandibular disorders are very diverse, so a detailed analysis of each case should be made to evaluate the possible concomitance of several of them, to give greater weight to the possible etiological factor and to pay attention to those that are Lasting or aggravating. An observational, descriptive, cross-sectional study was conducted to identify the risk factors associated with temporomandibular disorders. The universe was comprised of 70 patients who came to the consultation at the time of the investigation. The main variables analyzed were: age, sex, predisposing factors, precipitating factors, systemic diseases. In the series it was obtained that of the total of patients, 41 belonged to the female sex, to $58.5 \%$. The age group 40-49 was represented by 35 patients for $50.0 \%$. In $92.4 \%$, bruxism was observed, in $91.4 \%$, unilateral mastication was observed, while $92.8 \%$ presented missing teeth that had not been restored.

Keywords: temporomandibular disorders; risk factors; temporomandibular joint. 


\section{Resumo}

Os fatores de risco dos distúrbios temporomandibulares são muito diversos, portanto, uma análise detalhada de cada caso deve ser feita para avaliar a possível concomitância de vários deles, a fim de dar maior peso ao possível fator etiológico e prestar atenção aos que são duradoura ou agravante. Um estudo observacional, descritivo e transversal foi conduzido para identificar os fatores de risco associados a distúrbios temporomandibulares. O universo era composto por 70 pacientes que chegaram à consulta no momento da investigação. As principais variáveis analisadas foram: idade, sexo, fatores predisponentes, fatores precipitantes, doenças sistêmicas. $\mathrm{Na}$ série obteve-se a do total de pacientes, 41 pertenciam ao sexo feminino, a 58,5\%. A faixa etária 40-49 foi representada por 35 pacientes por 50,0\%. Em 92,4\% de bruxismo foi observado, em $91,4 \%$ de mastigação unilateral, enquanto $92,8 \%$ apresentaram dentes faltantes que não foram restaurados.

Palavras chave: distúrbios temporomandibulares; fatores de risco; articulação temporomandibular.

\section{Introducción}

La lucha contra las enfermedades constituye una constante en el desarrollo cultural del ser humano, esta constante ha permitido un incremento de la esperanza de vida, que ha dado lugar a los cambios demográficos que se expresan hoy, con un aumento de la población geriátrica, conocido como envejecimiento poblacional. ${ }^{1}$

El conocimiento del estado de salud de la población, con un enfoque clínico-epidemiológico y social, basada en un análisis de hallazgos a través de la situación de salud (ASS) resulta una premisa indispensable para el desarrollo de una planificación, organización, dirección y control de la atención médica odontológica. ${ }^{1}$

La salud bucodental es un constituyente inseparable e importante de la salud general del individuo y tiene consecuencias fisiológicas y sociales aún más complejas, porque puede llegar a menoscabar la nutrición, las relaciones interpersonales y la salud mental. Dentro de estas enfermedades podemos decir que (los desórdenes articulares son patologías consideradas dentomaxilofaciales y repercute en el organismo en general por la cantidad de síntomas y signos que presentan y a su vez no existe ningún signo por sí solo que pueda caracterizar la enfermedad. 
Hasta hace muy poco estas patologías eran consideradas como «tierra de nadie» en el ámbito profesional de las Ciencias Médicas. ${ }^{1}$

El sistema estomatognático (SE) es la unidad biológica o morfofuncional localizada anatómicamente en el territorio cráneo-cérvico-facial limitado por un plano horizontal superior que pasa a la altura de los rebordes supraorbitarios y uno inferior que pasa a la altura del hueso hiodes. Este sistema cumple una serie de funciones tales como: masticación, deglución y fonoarticulación; pero también cumple una importante participación en la respiración y la degustación. Este sistema se encuentra conformado por: un componente neuromuscular, un componente esquelético, ATM, oclusión dentaria y periodonto. Además existe un intrincado sistema de control neurológico que regula y coordina todos estos componentes estructurales. ${ }^{2}$

Los trastornos témporomandibulares (TTM) se pueden definir como un grupo de condiciones músculo-esqueletales que afectan a la articulación témporomandibular, a los músculos masticadores y a las estructuras asociadas, generando un conjunto de signos y síntomas, siendo los más relevantes el dolor articular, los ruidos articulares, las alteraciones de la dinámica mandibular y el dolor miofascial. Entre este conjunto de signos y síntomas, el dolor aparece como la principal causa de consultas y solicitudes de tratamiento. El dolor de tipo crónico asociado a los TTM representa una fuente de malestar y grave alteración de la calidad de vida de las personas que lo padecen, y enfrentarlo constituye un desafío para toda la profesión odontológica.$^{3}$.

Según Wagner de Oliveira, la etiología de la disfunción temporomandibular es uno de los asuntos más controvertidos y estudiados de la Odontología. Quizá porque no se encuentra una clara relación causa-efecto o porque faltan fundamentos científicos concretos pero, sin embargo, sí parece haber consenso en la comunidad científica de que se trata de un trastorno multifactorial que se desglosa en factores predisponentes que aumentan el riesgo de la aparición de un trastorno, factores desencadenantes que provocan en última instancia el comienzo de un trastorno y los factores perpetuantes que impiden la curación y favorecen el agravamiento. ${ }^{4}$

La teoría de los factores de riesgo es uno de los logros de la medicina contemporánea, que permite controlar aquellos elementos o procesos que al incidir negativamente obstaculizan la ulterior calidad de la salud de la población. Los factores no actúan de forma independiente, 
aislada, sino en conjunto, interrelacionados, fortaleciendo su nocivo efecto para la salud. Los factores pueden ser indicadores de riesgo como causa de daño a la salud. ${ }^{5,1}$

En cuanto a los factores de riesgo de la ATM se acepta que son múltiples, por lo que se considera multifactorial, y se hace necesario evaluar el sistema neuromuscular, oclusal, articular, y alteraciones a los movimientos, así como ciertos factores psicológicos y estilos de vida en la obtención de un diagnóstico correcto, a su vez para poder trazar una estrategia de intervención que impida el poder desencadenar la enfermedad, una vez que estos factores actúen como estímulo nocivos en el tiempo y sobre una estructura dada, se puede romper la capacidad adaptativa del individuo y aparece la enfermedad, pues el factor puede estar presente por tiempo y no desencadenarse las alteraciones de la articulación, pero el trabajo del odontólogo de la atención primaria es tratar de eliminar estos factores, evitando de esta forma que el individuo desencadene la enfermedad. ${ }^{1}$

Los factores de riesgo actúan por un tiempo determinado en un órgano; ejemplo de ello tenemos la interferencia oclusal, debido a una obturación en exceso actúa sobre el organismo, esto produce consecuencias negativas en el mismo, fundamentalmente sobre el sistema neuromuscular, en este tejido se produce una adaptación a la nueva situación que produce el estrés mantenido como agente nocivo sobre el tejido. El organismo da una respuesta (ruptura de la capacidad adaptativa del individuo al agente nocivo) la respuesta es una hipertonicidad muscular, contractura mantenida, esta trae como consecuencia desarmonías oclusales, buscando la mandíbula una nueva posición mandibular aliviando, o acomodándola a la desarmonía oclusal, esto en un tiempo dado trae aparejado limitaciones de los movimientos, o nuevos patrones de movimientos mandibulares, dolor, alteraciones musculares y articulares que a la vez agravan el sistema estomatognático y producen el circulo de retroalimentación con más alteraciones psicológicas, y por tanto una disfunción de la articulación temporomandibular. ${ }^{6,1}$

Es común ver a pacientes que sin considerar consecuencias se exponen constantemente a factores, que a continuación exponemos:

a. Masticar constantemente gomas (chicles).

b. Masticar alimentos duros (semillas de guayaba, granos de arroz, hielo, chicharrones, etc.) 
c. Realizar golpeteo constantemente entre dientes y dientes.

d. Apretar los dientes uno contra otro.

e. Masticar de un solo lado. (masticación unilateral en la arcada dentaria)

f. Bostezo exagerado.

g. Gritar al hablar de forma exagerada. (amplitud exagerada de cavidad bucal)

h. Accidentes golpeándose la mandíbula.

i. Hábitos posturales. (Apoyo de la cabeza de un solo lado, apoyo de la mandíbula sobre la mano, no mantener la cabeza erguida, cargar peso de un solo lado, etc.

j. Hábitos no funcionales. (onicofagia, queilofagia, etc.)

k. Estrés.

1. Estilos de vida no funcionales. ${ }^{1}$

Teniendo en cuenta lo anterior, los autores de este trabajo se propusieron identificar los factores de riesgos asociados a los trastornos temporomandibulares, para de esta forma trabajar sobre los problemas y los factores de riesgo y mejorar la calidad de vida de los pacientes, siendo esta la labor fundamental del equipo de salud.

\section{Material y método}

Se realizó estudio observacional, descriptivo, de corte transversal con vista a identificar los factores de riesgo asociados a los trastornos temporomandibulares.

El universo estuvo comprendido por 70 pacientes que acudieron a la consulta en el momento de la investigación, periodo 2015 que refirieron alguna dificultad en la articulación temporomandibular.

Como principales variables analizadas figuraron: edad, sexo, factores predisponentes: (adaquia, oclusión invertida, resalte aumentado, dientes ausentes no restituidos, prótesis defectuosas), factores precipitantes: (bruxismo, masticación unilateral, morder objetos) estrés, enfermedades sistémicas. 
La fuente de recolección de datos fueron las 70 historias clínicas de los pacientes con TTM, la consulta cuenta con un sillón odontológico y el instrumental y materiales necesarios como: espejo bucal, guantes, regla milimetrada y estetoscopio para realizarle a los pacientes el examen bucal, basado en la inspección de la cavidad bucal y de los movimientos mandibulares, así como la palpación y auscultación de la ATM.

El procesamiento estadístico-matemático de la información se realizó con el sistema SPSS versión 11.5, y el empleo del porcentaje como medida de resumen.

\section{Resultado y discusión}

Tabla 1. Distribución de pacientes con TTM según edad y sexo.

\begin{tabular}{|c|c|c|c|c|c|c|}
\hline \multirow[t]{2}{*}{ Edad } & \multicolumn{2}{|c|}{ Masculino } & \multicolumn{2}{|c|}{ Femenino } & \multicolumn{2}{|c|}{ Total } \\
\hline & No & $\%$ & No & $\%$ & No & $\%$ \\
\hline $20-29$ & 5 & 17.2 & 6 & 14.6 & 11 & 15.7 \\
\hline 30- 39 & 5 & 17.2 & 10 & 24.3 & 15 & 21.4 \\
\hline $40-49$ & 13 & 44.8 & 22 & 53.6 & 35 & 50.0 \\
\hline+50 & 6 & 20.6 & 3 & 7.3 & 9 & 12.8 \\
\hline Total & 29 & 41.4 & 41 & 58.5 & 70 & 100 \\
\hline
\end{tabular}

En la serie se observó un predominio de los pacientes en las edades de 40-49 años (50.0 \%), La incidencia de la enfermedad fue mayor en el sexo femenino, con $58.5 \%$. lo cual coincide con otras investigaciones, como son la realizada por Rodríguez Carracedo y otros ${ }^{7}$. Diversas teorías han tratado de explicar esta tendencia, algunas lo atribuyen a una mayor sensibilidad de las féminas ante los signos y síntomas de los TTM que las lleva a solicitar ayuda profesional. 
Tabla 2. Distribución de pacientes con TTM según factores predisponentes.

\begin{tabular}{|c|c|c|}
\hline Factores predisponentes & No de pacientes & No \\
\hline Oclusión invertida & 55 & 78.5 \\
\hline Resalte aumentado & 30 & 42.8 \\
\hline $\begin{array}{c}\text { Dientes ausentes no } \\
\text { restituidos }\end{array}$ & 65 & 92.8 \\
\hline Prótesis defectuosas & 62 & 88.5 \\
\hline Adaquia & 22 & 31.4 \\
\hline
\end{tabular}

Se observó un gran número de pacientes con factores predisponentes, especialmente de trastornos oclusales. La oclusión dentaria, condiciona la posición de los cóndilos en la cavidad glenoidea de tal forma, que anomalías de la oclusión, incluso algunas sutiles, pueden provocar problemas articulares importantes. Los dientes ausentes no restituidos protésicamente, con el paso del tiempo conllevan a una reabsorción ósea, que deriva en una disminución de la dimensión vertical y repercute en el estado neuromuscular, propioceptivo y postural, quedando a expensas de la capacidad de adaptación de cada persona, lo que reporta una gran variabilidad de respuesta y predispone al individuo a presentar TTM. Taboada ${ }^{8}$, plantea que los TTM se agravan ante la evidencia de los efectos nocivos que tiene la falta de sustitución protésica inmediata a la pérdida dental. Como se aprecia en la tabla 2 , manifiesta que entre los pacientes que presentaban factores predisponentes de TTM, 65 para un $92.8 \%$ tenían dientes ausentes no restituidos, seguidos de 62 con prótesis desajustadas para un $88.5 \%$, los resultados coinciden con el estudio realizado por de la Torre Rodríguez ${ }^{9}$. Otro estudio realizado por Martínez Brito ${ }^{10}$, el $41.9 \%$ de los pacientes con trastornos en la articulación temporomadibular presentaban perdida dentaria.

Los factores de riesgo odontológicos producen nuevos patrones de movimientos mandibulares y posiciones mandibulares limitando la función normal de la ATM, que a la vez estas deben ser de interés de los odontólogos debido al daño que pueden producir en los pacientes. ${ }^{1}$ 
La relación intrínseca entre alteración oclusal y trastorno articular no es un paradigma que se cumpla siempre. Sin embargo, el análisis oclusal debe ser siempre un requisito imprescindible en la exploración del paciente disfuncional ya que multitud de estudios demuestran que puede representar un factor etiológico de primer orden. ${ }^{11}$

Tabla 3. Distribución de pacientes con TTM según factores precipitantes.

\begin{tabular}{|c|c|c|}
\hline Factores precipitantes & No de pacientes & No \\
\hline Macrotrauma facial & 22 & 31.4 \\
\hline Bruxismo & 66 & 92.4 \\
\hline Masticación unilateal & 64 & 91.4 \\
\hline Morder objeto & 20 & 28.5 \\
\hline
\end{tabular}

Entre los pacientes que presentaban factores precipitantes de TTM, 66 padecían bruxismo, lo que figura un $92.4 \%$ del total, seguido de 64 pacientes que presentaban masticación unilateral (91.4\%), como lo demuestra la tabla 3.

Las variables que generan presión intraarticular, como el estrés $(87.1 \%)$, que por lo general se manifiesta en los pacientes con bruxismo y la masticación unilateral, que también produce sobrecarga en una de las articulaciones, mostraron valores porcentuales elevados.

El estrés emocional puede influir también en los síntomas de los TTM, ya que puede reducir la tolerancia fisiológica del paciente. Ello se debe probablemente a un incremento del tono simpático, efecto que suele representar la respuesta aprendida del individuo ante diferentes factores estresantes. Esta respuesta simpática aprendida ante el estrés, desempeña un papel destacado en el dolor crónico. ${ }^{12}$

Las enfermedades sistémicas juegan un papel importante entre los factores de riesgo biológicos que se relacionan con los TTM. En la serie, solo un paciente presentaba como diagnostico artritis reumatoide.

En la bibliografía consultada se hallaron estudios similares los cuales, consideran importante identificar la existencia de determinados factores de riesgo que puedan producir daño en el funcionamiento de la articulación temporomandibular. 
Se llegaron a las conclusiones que el sexo predominante fue el femenino y el grupo de edades más representado el de 40-49 años. El factor predisponerte que más se observó fueron los dientes ausentes no restituidos protésicamente.

\section{Referencias bibliográficas}

1. Alfonso Adán X, Renda Valera L. Factores de riesgo que actúan sobre la articulación temporomandibular. Universidad de Ciencias Médicas de La Habana. Instituto de Ciencias Básicas y Preclínicas «Victoria de Girón» Disponible en:www.sld.cu/.../factores_de_riesgo_que_actuan_sobre_la_articulacion_temporomandi

2. Okeson JP. Tratamiento de oclusión y afecciones temporomandibulares.2013 7ed. Elsevier, pp. 233-242.

3. Romo F, Díaz W, Schulz R, Torres M. Tópicos de odontología integral.2011 Santiago, Chile: Universidad de Chile

4. Oliveira W: Disfunções Temporomandibulares. Sao Paulo: Artes Médicas; 2002:135.

5. Aguilar M. Trastornos de la articulación temporomandibular. México: 2003. Disponible en: http/www.com.ar/articulo-central.htm

6. America dental Association. 2004. Disponible en: tmp/tmj.http:/www.ada org/public/topics/tmo-tmj.osp.

7. Rodríguez EM, Díaz JE, Carmona E, Segura O, Pellitero B, Carracedo P. Prevalencia de los trastornos temporomandibulares en adolescentes con maloclusiones. ESBU «Juan José Fornet». Holguín, 2005. Correo Científico Médico de Holguín. 2007 [citado 18 ene 2017]; 11(1). Disponible en: http://www.cocmed.sld.cu/no111/n111ori1.htm

8. Taboada O, Gómez Y, Gutiérrez, Taboada S, Mendoza VM. Prevalencia de signos y síntomas de los trastornos temporomandibulares en un grupo de adultos mayores. Rev ADM. 2004; 61(4).

9. De la Torre Rodríguez E, Aguirre Espinosa I, Fuentes Mendoza V, D Peñón Vivas P A, Espinosa Quirós D, Núñez Fernández Y. Factores de riesgo asociados a trastornos temporomandibulares. Revista cubana de estomatología 2013 [citado 18 ene 2017]; 
50(4). Disponible en:

http://www.revestomatologia.sld.cu/index.php/est/article/view/225/42

10. Martinez Brito, I et al. Factores de riesgo en pacientes con disfunción temporomandibular. Rev. Med. Electrón. 2009 [citado 18 ene 2017]; vol.31, n.4, pp. 0-0. Disponible en: http://scielo.sld.cu/scielo.php?script=sci_abstract\&pid=S1684-18242009000400004

11. García-Fajardo Palacios Carlos, Cacho Casado Alberto, Fonte Trigo Abelardo, Pérez Varela Juan Carlos. La oclusión como factor etiopatológico en los trastornos temporomandibulares. RCOE. 2007 [citado 2016 Diciembre 16]; 12(1-2): 37-47. Disponible en: http://scielo.isciii.es/scielo.php?script=sci_arttext\&pid=S1138$123 \times 2007000100003 \& \operatorname{lng}=\mathrm{es}$.

12. Cabo García R1 Grau León I B 1 Lorenzo Uribazo A M 1 Factores de riesgo de los trastornos temporomandibulares en el adulto mayor. Medisur 2016. 14(2). 